\title{
A Class of Highly Concentrated Time-Frequency Distributions Based on the Ambiguity Domain Representation and Complex-Lag Moment
}

\author{
Irena Orović and Srdjan Stanković \\ Electrical Engineering Department, University of Montenegro, 81000 Podgorica, Montenegro \\ Correspondence should be addressed to Irena Orović, irenao@cg.ac.yu
}

Received 3 October 2008; Revised 13 April 2009; Accepted 9 June 2009

Recommended by Y.-P. Lin

A class of time-frequency distributions with complex-lag argument is proposed. It is based on the ambiguity domain representations of real and complex-lag moment, combined to provide a cross-terms free representation for multicomponent signals. Furthermore, the distributions from the proposed class provide a more effective instantaneous frequency estimation for signals with fast varying phase function than the existing approach. The theory is illustrated by the examples.

Copyright (c) 2009 I. Orović and S. Stanković. This is an open access article distributed under the Creative Commons Attribution License, which permits unrestricted use, distribution, and reproduction in any medium, provided the original work is properly cited.

\section{Introduction}

In order to provide an efficient instantaneous frequency estimation for nonstationary signals, the time-frequency analysis has attracted the attention of many researchers. Depending on the applications, various time-frequency distributions have been proposed. Among them, commonly used, are quadratic distributions from the Cohen class $[1,2]$. It is well known that the Wigner distribution, for example, provides an ideal concentration along the linear instantaneous frequency. However, it suffers from cross terms in the case of multicomponent signals. Also, for nonlinear instantaneous frequency, the inner interferences appear. Thus, for the analysis of signals with varying instantaneous frequency, higher-order distributions are used [3-7]. Also, some interesting methods based on neural networks $[8,9]$, sparsity constraint of energy distribution [10], and autoregressive moving-average models [11] have been recently introduced to improve the resolution in the time-frequency domain.

In order to provide an accurate instantaneous frequency estimation even when the signal phase varies significantly within a few signal samples, the distributions with complexlag argument have been introduced [12-15]. However, it is still difficult to deal with cross-terms in the case of multicomponent signals. The problem may also appear in the numerical calculation of the analytic extension that is used to obtain signal with complex-valued argument. It might affect the precision of instantaneous frequency estimation. A solution for multicomponent signals has been proposed in [16]. Namely, the Nth order complex-lag distribution is obtained by convolving the $S$-method with the concentration function (of order $N-2$ ) in the time-frequency plane. This approach introduces significant approximations that cause additional complexity in the numerical realization.

In this paper we propose a new class of time-frequency distributions whose members can provide an efficient estimation of fast varying instantaneous frequency for multicomponent signals. This concept is established in the ambiguity domain, and it can be seen as a more effective counterpart of the method in [16]. Starting from the definition of the generalized complex-lag time-frequency distribution [15], two ambiguity domain representations are defined. The first one is related to the signal with real-valued argument and corresponds to the standard ambiguity function. The second one is related to the signal terms with complex-valued argument. In order to obtain a highly concentrated representation, free of cross-terms, inner 
interferences, and other disturbances, the two ambiguity domain representations are filtered by using the suitable kernel function. Afterwards, the resulting generalized ambiguity domain representation is obtained by performing $2 \mathrm{D}$ convolution within the ambiguity domain. A new form of the $N$ th order complex-lag time-frequency distribution follows as a two-dimensional inverse Fourier transform of the proposed ambiguity domain representation. More generally, by considering various existing kernels, the entire class of complex-lag distributions is defined. Thus, instead of using the $S$-method as in [16], various distributions from the Cohen class can be used. The theoretical considerations are illustrated by the examples.

\section{Theoretical Background-Complex-Lag Time-Frequency Distribution}

The distribution with complex-lag argument has been introduced to provide a highly concentrated representation along the instantaneous frequency for signals with fast varying phase function. The complex time-frequency distribution has been defined in the form [12]

$$
\begin{aligned}
\operatorname{CTD}(t, \omega)= & \int_{-\infty}^{\infty} x\left(t+\frac{\tau}{4}\right) x^{*}\left(t-\frac{\tau}{4}\right) \\
& \times x^{-j}\left(t+j \frac{\tau}{4}\right) x^{j}\left(t-j \frac{\tau}{4}\right) e^{-j \omega \tau} d \tau .
\end{aligned}
$$

This distribution satisfies a number of important properties $[12,13]$ : it is real-valued (for $x(t)=r e^{j \phi(t)}$ ), time-shift, and frequency-shift covariant, satisfying marginal properties, time-frequency scaling property, and so forth. Also, it significantly reduces the spread factor compared to the quadratic distributions [12] (meaning the concentration improvement): $Q(t, \tau)=\phi^{(5)}(t) \tau^{5} /\left(4^{4} 5 !\right)+\phi^{(9)}(t) \tau^{9} /\left(4^{8} 9 !\right)+$ $\ldots$. Note that the dominant term within spread factor $Q(t, \tau)$ is of the fifth order, while in the case of Wigner distribution this term is of the third order.

The values of signal with complex-lag argument $x(t+j \tau)$ are calculated by using the analytic extension of $x(t)$ as follows:

$$
x(t+j \tau)=\int_{-\infty}^{\infty} X(\omega) e^{-\tau \omega} e^{j \omega t} d \omega
$$

where $X(\omega)$ is the spectrum of $x(t)$.

Further improvement of the concentration in the timefrequency domain is obtained by increasing the distribution order. Therefore, the generalization of complex-lag distribution for signals with fast varying instantaneous frequency has been introduced $[15,16]$ :

$$
\operatorname{GCD}(t, \omega)=\int_{-\infty}^{\infty} x\left(t+\frac{\tau}{N}\right) x^{*}\left(t-\frac{\tau}{N}\right) M_{c}(t, \tau) e^{-j \omega \tau} d \tau .
$$

The complex-lag signal moment $M_{c}(t, \tau)$ is defined as [16]

$$
M_{c}(t, \tau)=\prod_{k=1}^{N / 2-1} x^{w_{N, k}^{*}}\left(t+\frac{w_{N, k}}{N} \tau\right) x^{-w_{N, k}^{*}}\left(t-\frac{w_{N, k}}{N} \tau\right),
$$

where $w_{N, k}=e^{j 2 \pi k / N}$ defines the roots on the unit circle, while $N$ is an even number representing the distribution order. Observe that the real-lag signal moment $M_{r}(t, \tau)=$ $x(t+\tau / N) x^{*}(t-\tau / N)$ leads to the Wigner distribution (special case for $N=2$ ), while the complex-lag moment represents the concentration function whose number of terms depends on the distribution order. Unlike the Wigner distribution, the generalized complex-lag time-frequency distribution of order $N \geq 4$ provides an arbitrary high concentration even when the variations of instantaneous frequency are within a few samples. The spread factor is given by

$$
Q(t, \tau)=\frac{\phi^{(N+1)}(t) \tau^{N+1}}{N^{N}(N+1) !}+\frac{\phi^{(2 N+1)}(t) \tau^{2 N+1}}{N^{2 N}(2 N+1) !}+\cdots
$$

Thus, the distribution spread factor can be arbitrarily reduced by the appropriate choice of $N$.

Note that the generalized complex-lag time-frequency distribution preserves the properties of the distribution defined by (1), which is a special case for $N=4$. In the case $N$ $=6$, the complex roots $w_{N, k}$ on the unit circle are defined as: $w_{6,1}=\sqrt{3} / 2+\mathrm{j}(1 / 2), w_{6,2}=\sqrt{3} / 2-\mathrm{j}(1 / 2)$, and the following form is obtained:

$$
\begin{aligned}
\operatorname{GCD}(t, \omega)=\int_{-\infty}^{\infty} & x\left(t+\frac{\tau}{6}\right) x^{*}\left(t-\frac{\tau}{6}\right) \\
& \times x^{w_{6,1}^{*}}\left(t+w_{6,1} \frac{\tau}{6}\right) x^{-w_{6,1}^{*}}\left(t-w_{6,1} \frac{\tau}{6}\right) \\
& \times x^{w_{6,2}^{*}}\left(t+w_{6,2} \frac{\tau}{6}\right) x^{-w_{6,2}^{*}}\left(t-w_{6,2} \frac{\tau}{6}\right) e^{-j \omega \tau} d \tau .
\end{aligned}
$$

Although the generalized complex-lag time-frequency distribution represents an efficient tool for the instantaneous frequency analysis, the presence of cross-terms in the case of multicomponent signals is still a challenging problem. In this case, the significant approximations are needed [16], which consequently affect the properties of the distribution (e.g., marginal properties are satisfied only when a single component is observed). An additional problem appears in the numerical realization, which is again especially emphasized for multicomponent signals. Namely, in the calculation of signal with complex-lag argument, the real exponential function $e^{-\tau \omega}$ assumes large values as $\tau$ increases, leading to the serious miscalculations due to the software precision range. These calculation errors affect the accuracy of the instantaneous frequency estimation, especially in the case of rapid phase variations when larger $N$ is preferred.

\section{A Class of Complex-Lag Time-Frequency Distributions}

3.1. Ambiguity Domain Representations of Real and ComplexLag Moment. In order to overcome the difficulties in the case of multicomponent signals, the idea is to use the advantages of the ambiguity domain, not only to provide cross-terms free time-frequency representation, but also to efficiently remove disturbances caused by the miscalculation of analytic 
extension. Moreover, the complex-lag distribution in [16] will be extended to a class of complex-lag distributions. In this sense, the proposed approach aims to keep good properties of Cohen class distributions within a class of complex-lag distributions.

The ambiguity function related to the real-lag moment can be written as

$$
\begin{aligned}
\operatorname{AF}_{r t}(\theta, \tau) & =\int_{-\infty}^{\infty} M_{r}(t, \tau) e^{-j \theta t} d t \\
& =\int_{-\infty}^{\infty} x\left(t+\frac{\tau}{N}\right) x^{*}\left(t-\frac{\tau}{N}\right) e^{-j \theta t} d t
\end{aligned}
$$

It corresponds to the standard ambiguity function: $\operatorname{AF}_{r t}(\theta, \tau)=\operatorname{AF}(\theta, 2 \tau / N)$, with the autoterms located around the origin, while the cross-terms, in the case of multicomponent signals, are dislocated from the auto-terms.

In analogy with (7), we can also define the ambiguity domain representation of the complex-lag moment $M_{c}(t, \tau)$ as follows:

$$
\begin{aligned}
& \operatorname{AF}_{c t}(\theta, \tau) \\
& \quad=\int_{-\infty}^{\infty} M_{c}(t, \tau) e^{-j \theta t} d t \\
& \quad=\int_{-\infty}^{\infty} \prod_{k=1}^{N / 2-1} x^{w_{N, k}^{*}}\left(t+\frac{w_{N, k}}{N} \tau\right) x^{-w_{N, k}^{*}}\left(t-\frac{w_{N, k}}{N} \tau\right) e^{-j \theta t} d t .
\end{aligned}
$$

The signal's terms in (8) are obtained by using the analytic extension of $x(t)$. In the case of multicomponent signals it can be calculated as follows:

$$
\begin{aligned}
x( & \left.t \pm \frac{w_{N, k}}{N} \tau\right) \\
& =\sum_{m=1}^{M} x_{m}\left(t \pm \frac{w_{N, k}}{N} \tau\right) \\
& =\sum_{m=1}^{M} \int_{-W_{m}}^{W_{m}} \operatorname{STFT}\left(t, \omega+\omega_{m}(t)\right) e^{j\left(\omega+\omega_{m}(t)\right)\left(t \pm w_{N, k}(\tau / N)\right)} d \omega,
\end{aligned}
$$

where $M$ is the number of signal components, $2 W_{m}$ is the width of signal component, while $\omega_{m}(t)=$ $\arg \left\{\max _{\omega} \operatorname{STFT}(t, \omega)\right\}$. Note that during the computation of the analytic extension, the miscalculations may appear for large values of $\omega \tau$, that should be filtered within the $\mathrm{AF}_{c t}(\theta, \tau)$.

In order to remove cross-terms and miscalculation disturbances from the two proposed ambiguity domain representations, a kernel function $C(\theta, \tau)$ is considered. The filtered ambiguity domain representations are obtained as:

$$
\begin{aligned}
& \operatorname{AF}_{r t}^{c}(\theta, \tau)=C(\theta, \tau) \mathrm{AF}_{r t}(\theta, \tau), \\
& \operatorname{AF}_{c t}^{c}(\theta, \tau)=C(\theta, \tau) \mathrm{AF}_{c t}(\theta, \tau) .
\end{aligned}
$$

TABLE 1: Some of the commonly used kernels.

\begin{tabular}{lr}
\hline Choi-Williams kernel & $C(\theta, \tau)=e^{-\theta^{2} \tau^{2} / \sigma}, \sigma>0$ \\
Born-Jordan kernel & $C(\theta, \tau)=\frac{\sin (\theta \tau / 2)}{\theta \tau / 2}$ \\
Cone-kernel & $C(\theta, \tau)=e^{-\alpha \tau^{2}} \frac{\sin (\theta \tau / 2)}{\theta \tau / 2}$ \\
Sinc kernel & $C(\theta, \tau)=\operatorname{rect}(\theta \tau, \alpha)$ \\
\hline
\end{tabular}

Since $\operatorname{AF}_{r t}(\theta, \tau)$ contains all information about signal's component, the kernel could be adjusted to the auto-terms of $\operatorname{AF}_{r t}(\theta, \tau)$, as in the case of Cohen class derivation [17, 18]. Some of the signal independent kernels are given in Table 1. The commonly used fixed kernels have a number of desirable properties. For example, time-shift and frequencyshift properties are satisfied; the realness is satisfied if $C(\theta, \tau)=C^{*}(-\theta,-\tau)$, while the marginal properties: signal instantaneous power and power density spectrum are satisified if $C(\theta, 0)=1$ and $C(0, \tau)=1$, respectively. One might observe, for instance, that the Choi-Williams, Born-Jordan, and Sinc kernel satisfy both marginal properties, unlike the Cone-kernel that satisfies only the signal instantaneous power. Generally, the properties of the distributions from the proposed class will depend on the kernel, as in the case of Cohen class distribution.

In many cases, the kernels show a preferred behavior in the time and/or the frequency direction. The degree of cross-terms reduction depends on the way in which the Wigner distribution is oriented in the time-frequency plane. Usually, there is a trade-off between cross-terms suppression and auto-terms concentration $[19,20]$. The cross-terms attenuation in the case of multicomponent signals is highly sensitive to the relative time-frequency location of interfering signal components [21]. Namely, if signal components occur either around the same time or around the same frequency, the interference terms are located around $\tau$ or $\theta$ axis. In this case, the shape of fixed kernels from Table 1 cannot provide removal of cross-terms without affecting the auto-terms.

In the examples, we have also considered the fixed Gaussian kernel: $C(\theta, \tau)=e^{-\left(\theta^{2}+\tau^{2}\right) / 2 \sigma^{2}}$. It satisfies marginal properties only when a single component is observed. However, the shape of this kernel provides the efficient removal of interference terms, without disturbing autoterms in the ambiguity domain, as it will be shown later. Also, the disturbances caused by the miscalculations of the analytic extension in $\operatorname{AF}_{c t}(\theta, \tau)$ are eliminated by using this kernel.

Note that various adaptive and signal dependent kernels could also be successfully used [20,22-26], such as Radially Gaussian kernel [22]. However, it is outside the scope of this paper and could be a topic of the future work.

3.2. Generalized Ambiguity Domain Representation and the Corresponding Class of Time-Frequency Distributions. As it was discussed in the previous section, in the case of highly 


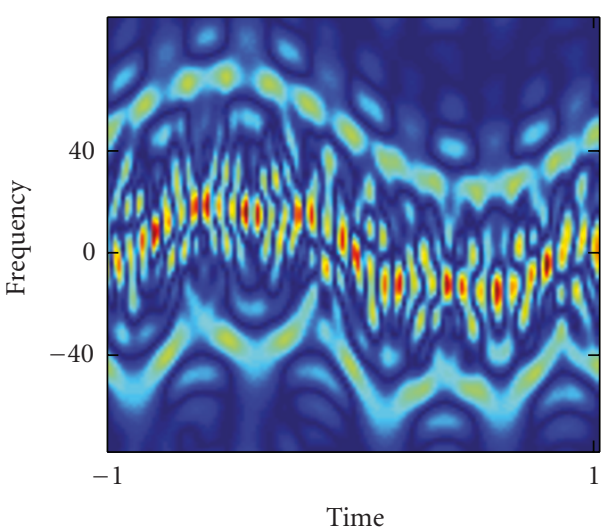

(a)

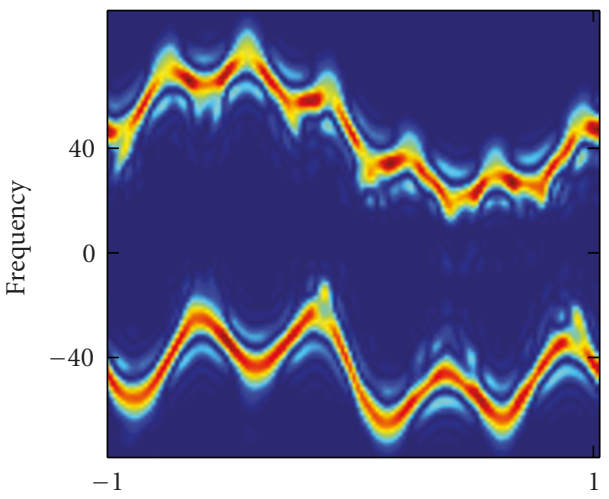

Time

(c)

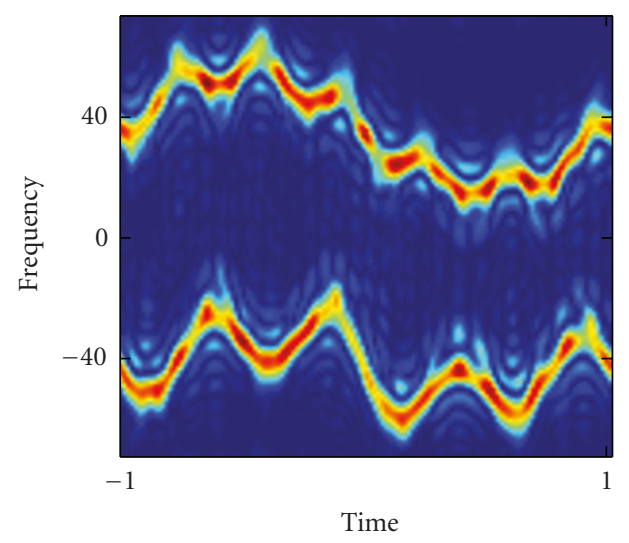

(e)

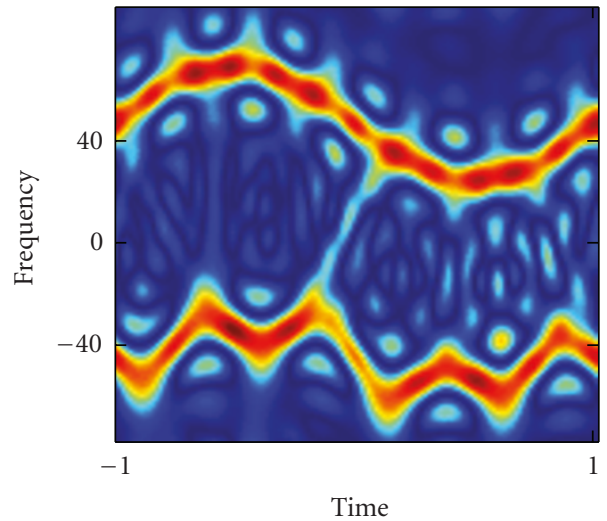

(b)

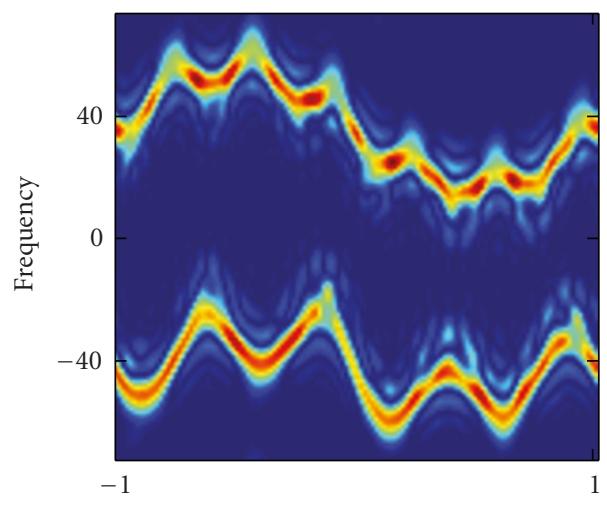

Time

(d)

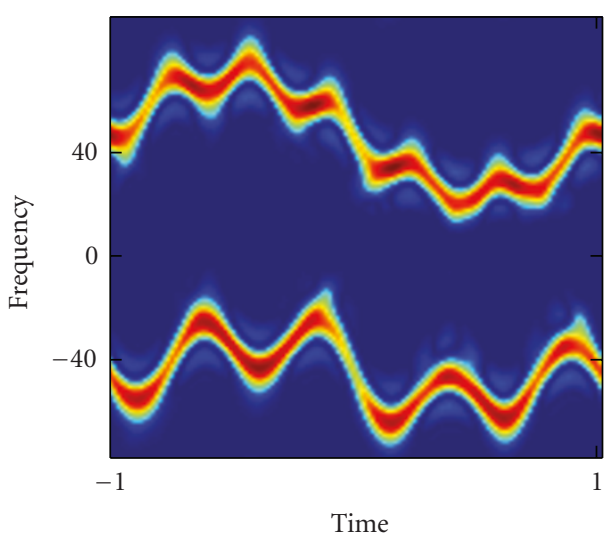

(f)

FIgURe 1: (a) Wigner distribution, (b) Wigner distribution with Gaussian kernel, (c) $\mathrm{GCD}_{\mathrm{AF}}(\mathrm{N}=4)$ with Choi-Williams kernel, (d) GCD $\mathrm{AF}_{\mathrm{F}}$ $(\mathrm{N}=4)$ with Born-Jordan kernel, (e) $\mathrm{GCD}_{\mathrm{AF}}(\mathrm{N}=4)$ with Sinc kernel, and (f) $\mathrm{GCD}_{\mathrm{AF}}(\mathrm{N}=4)$ with Gaussian kernel.

nonstationary signals, the concentration of the Wigner distribution is improved by the concentration function within the complex-lag distribution. Similarly, in the ambiguity domain, the $\operatorname{AF}_{c t}^{c}(\theta, \tau)$ (based on the complex-lag moment) acts as a correction term that improves the concentration of auto-terms in $\operatorname{AF}_{r t}^{c}(\theta, \tau)$. Thus, the resulting ambiguity domain representation for highly nonstationary signals can be obtained as a two-dimensional convolution of ambiguity functions $\operatorname{AF}_{r t}^{c}(\theta, \tau)$ and $\operatorname{AF}_{c t}^{c}(\theta, \tau)$. However, when dealing with multicomponent signals, it is necessary to introduce an additional window function to avoid new cross-terms that will appear due to convolution. Thus, following the analogy from the time-frequency domain, where the concept of windowing the product in convolution, has been introduced to define the S-method, that is, cross-terms free WD [27]:

$$
\operatorname{SM}(t, \omega)=\frac{1}{\pi} \int_{-\infty}^{\infty} P(\lambda) \operatorname{STFT}(t, \omega+\lambda) \operatorname{STFT}(t, \omega-\lambda) d \lambda,
$$




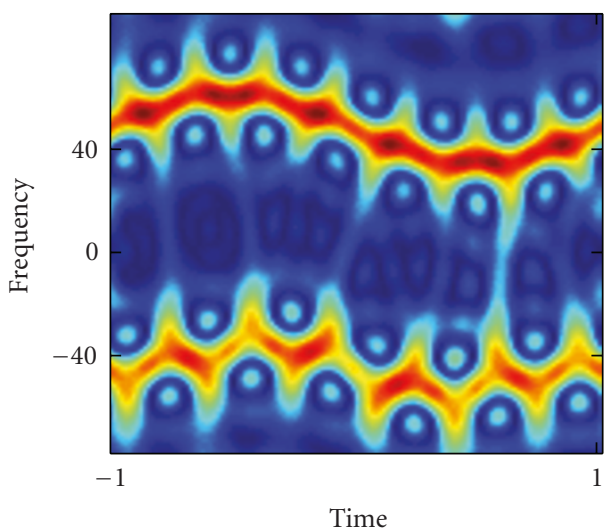

(a)

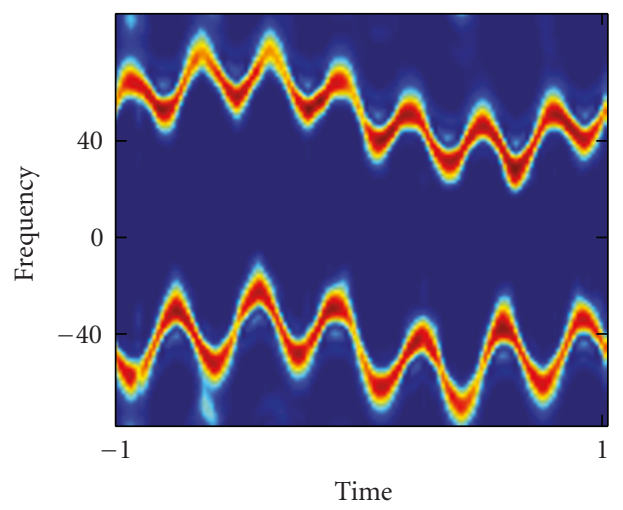

(c)

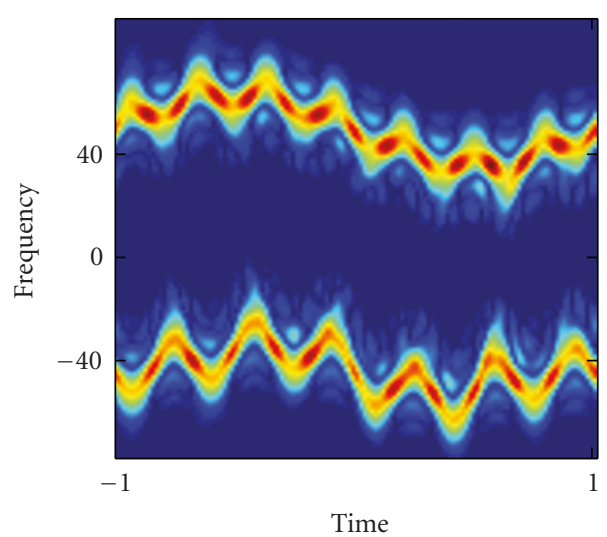

(b)

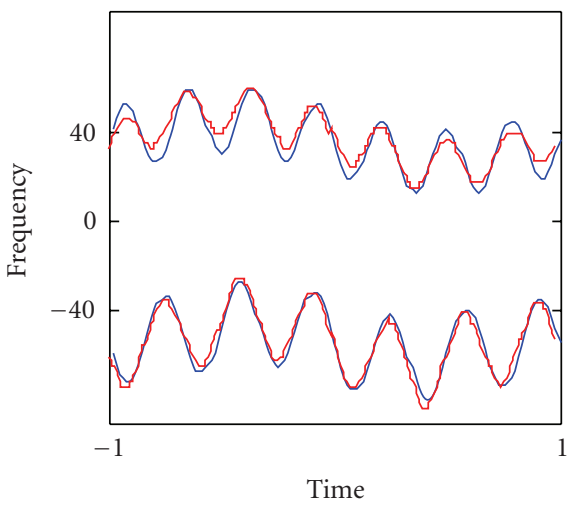

(d)

Figure 2: The complex time-frequency distributions from the proposed class for: (a) $N=2$; (b) $N=4$; (c) $N=6$; (d) the exact instantaneous frequency (blue line) and estimated instantaneous frequency from $\mathrm{GCD}_{\mathrm{AF}}, N=6$.

$(P(\lambda)$ is a frequency domain window), the resulting cross terms free ambiguity domain representation can be defined as:

$$
\begin{aligned}
\operatorname{AF}_{\mathrm{CTD}}(\theta, \tau)= & \iiint_{-\infty}^{\infty} P(\xi) e^{-j \xi \tau_{1}} e^{j \xi\left(\tau-\tau_{1}\right)} \\
& \times \operatorname{AF}_{r t}^{c}\left(\theta_{1}, \tau_{1}\right) \mathrm{AF}_{c t}^{c}\left(\theta-\theta_{1}, \tau-\tau_{1}\right) d \tau_{1} d \theta_{1} d \xi
\end{aligned}
$$

The cross-terms will be completely avoided if the size of the window $P(\xi)$ is less than the minimal distance between the auto-terms. The exponential functions in (12) correspond to the frequency shift in time-frequency domain. Based on the ambiguity domain representation, the new class of complex-lag time-frequency distributions is defined as follows:

$$
\operatorname{GCD}_{\mathrm{AF}}(t, \omega)=\frac{1}{2 \pi} \iint_{-\infty}^{\infty} \operatorname{AF}_{\mathrm{CTD}}(\theta, \tau) e^{j \theta t-j \omega \tau} d \tau d \theta
$$

3.2.1. Calculation Complexity. Observe that the proposed distribution for $N=2$ corresponds to quadratic distributions from the Cohen class. For higher order $N=$ $4,6, \ldots$, the calculation complexity increases due to the computation of complex-lag ambiguity function $\operatorname{AF}_{\mathrm{ct}}(\theta, \tau)$ (of order $N-2$ ). Comparing with the Cohen class distributions (e.g., Choi-Williams distribution), additional computations are related to $\operatorname{AF}_{c t}(\theta, \tau)$ and resulting ambiguity function (12). The calculation of $\mathrm{AF}_{c t}(\theta, \tau)$ involves calculation of signal with complex-lag argument $(N-2$ FFT routines $\left(\mathrm{O}\left(N_{s}(N-2) \log _{2} N_{s}\right)\right.$ operations for $N_{s}$ samples within the window), $M(N-2)$ IFFT routines by using $2 W_{m}$ samples $\left(\mathrm{O}\left(M(N-2) W_{m} \log _{2} 2 W_{m}\right)\right.$ operations), and $N_{s}$ complex multiplications with exponential terms); calculation of complex-lag signal moment $\left((N-3) N_{s}\right.$ complex multiplications), and FFT routine to obtain ambiguity function $\left(\mathrm{O}\left(N_{s} \log _{2} N_{s}\right)\right)$. Hence, the calculation of $\operatorname{AF}_{c t}(\theta, \tau)$ function is $\mathrm{O}\left((N-2) N_{s} \log _{2} N_{s}\right)$.

Filtering with kernel function: $\operatorname{AF}_{c t}^{c}(\theta, \tau)=C(\theta$, $\tau) \mathrm{AF}_{c t}(\theta, \tau)$ requires the same number of arithmetic operations as $\operatorname{AF}_{r t}^{c}(\theta, \tau)=C(\theta, \tau) \operatorname{AF}_{r t}(\theta, \tau)$, that is also used in the Cohen class distributions (e.g., Choi-Williams distribution). The resulting ambiguity function is obtained as a correlation within the window of length $2 L+1\left(N_{s}(2 L+2)\right.$ complex multiplications and $L \cdot N_{s}$ complex additions $\left(\mathrm{O}\left(N_{s}\right)\right)$. Assuming that the same circuit could be used for filtering of real and complex-lag ambiguity function, the proposed distribution for $N \geq 4$ results in $\mathrm{O}\left((N-2) N_{s} \log _{2} N_{s}\right)$ additional arithmetic operations compared to the ChoiWilliams distribution. 
3.3. Group Delay. For the purpose of group delay analysis, the frequency domain form of time-frequency distributions is used. Thus, in the sequel, the proposed class of timefrequency distributions will be considered in frequency domain, as well.

For a signal $X(\omega)=A(\omega) e^{j \varphi(\omega)}$, that represents the Fourier transform of $x(t)$, the real and complex-argument moments are defined as follows:

$$
\begin{aligned}
& M_{r}(\omega, \theta)=X\left(\omega+\frac{\theta}{N}\right) X^{*}\left(\omega-\frac{\theta}{N}\right) \\
& M_{c}(\omega, \theta)=\prod_{k=1}^{N / 2-1} X^{w_{N, k}^{*}}\left(\omega+\frac{w_{N, k}}{N} \theta\right) X^{-w_{N, k}^{*}}\left(\omega-\frac{w_{N, k}}{N} \theta\right) .
\end{aligned}
$$

Note that the signal with complex argument is obtained as:

$$
\begin{aligned}
X\left(\omega \pm \frac{w_{N, k}}{N} \theta\right) & =\frac{1}{2 \pi} \int_{-\infty}^{\infty} x(t) e^{\mp j w_{N, k} t / N} e^{-j \omega t} d t \\
& =\frac{1}{2 \pi} \int_{-\infty}^{\infty} x(t) e^{ \pm b t / N} e^{-j(\omega \pm a) t} d t \\
& \text { where } w_{N, k}=a+j b .
\end{aligned}
$$

The corresponding ambiguity functions related to the real and complex-argument moment are given by:

$$
\begin{aligned}
& A F_{r t}(\theta, \tau)=\frac{1}{2 \pi} \int_{-\infty}^{\infty} M_{r}(\omega, \theta) e^{j \omega \tau} d \omega \\
& A F_{c t}(\theta, \tau)=\frac{1}{2 \pi} \int_{-\infty}^{\infty} M_{c}(\omega, \theta) e^{j \omega \tau} d \omega .
\end{aligned}
$$

The resulting ambiguity function $A_{\mathrm{CTD}}(\theta, \tau)$ and the corresponding class of complex-argument time-frequency distributions are of the same form given by (12) and (13), respectively.

\section{Examples}

Depending on the rate of the instantaneous frequency variations within the window, distributions of various orders should be used. Namely, the spectrogram suffices for a constant instantaneous frequency, while for a linear instantaneous frequency one should use quadratic distributions. However, for faster variations of the instantaneous frequency within the window such that the presence of its second derivative, (i.e., third-phase derivative) is significant, one should use the complex-lag distribution with $N=4$. Further, if the presence of the fifth-phase derivative is also significant, the order $N=6$ should be used, and so forth. Having in mind physical properties of an observed signal generating process, one may have a rough idea about the rate of the IF variations, which may be used for the choice of an appropriate distribution order.

In the following examples, we deal with highly non-

\begin{tabular}{|c|c|}
\hline Distribution & MSE \\
\hline $\begin{array}{l}\text { Choi-Williams } \\
\text { distribution }\end{array}$ & 47 \\
\hline $\begin{array}{l}\mathrm{GCD}_{\mathrm{AF}} \text { for } N=2 \text {, that is, } \\
\text { Wigner distr. smoothed } \\
\text { with Gaussian kernel }\end{array}$ & 24 \\
\hline $\begin{array}{l}\mathrm{GCD}_{\mathrm{AF}} \text { for } N=4, \\
\text { Choi-Williams kernel }\end{array}$ & 11.9 \\
\hline $\begin{array}{l}\mathrm{GCD}_{\mathrm{AF}} \text { for } N=4 \text {, Sinc } \\
\text { kernel }\end{array}$ & 12.3 \\
\hline $\begin{array}{l}\mathrm{GCD}_{\mathrm{AF}} \text { for } N=4, \\
\text { Born-Jordan kernel }\end{array}$ & 13.16 \\
\hline $\begin{array}{l}\mathrm{GCD}_{\mathrm{AF}} \text { for } N=4 \text {, } \\
\text { Gaussian kernel }\end{array}$ & 9.5 \\
\hline
\end{tabular}
stationary multicomponent signals whose instantaneous frequency varies significantly within a few samples. They are periodically modulated and, in real cases, correspond to the radar signals generated by nonuniform rotation of reflecting point.
TABLE 2: MSE of the IF estimation.

TABLE 3: MSE of the IF estimation in the presense of noise.

\begin{tabular}{lcccc}
\hline Distribution & $\mathrm{SNR}=30 \mathrm{~dB}$ & $\mathrm{SNR}=20 \mathrm{~dB}$ & $\mathrm{SNR}=10 \mathrm{~dB}$ & $\mathrm{SNR}=5 \mathrm{~dB}$ \\
\hline $\mathrm{GCD}_{\mathrm{AF}}(t, \omega)$, & 10.49 & 13.01 & 18.15 & 29.75 \\
$N=4$ & & & & \\
$\begin{array}{l}\text { Choi- } \\
\text { Williams }\end{array}$ & 48.4 & 73.85 & 81.54 & 123.14 \\
distr. & & & & \\
\hline
\end{tabular}

Example 1. Consider the multicomponent signal in the form

$$
\begin{array}{r}
x(t)=\exp \left(j \cdot \left(\cos (4 \cdot \pi \cdot t)+\frac{2 \cos (6 \cdot \pi \cdot t)}{3}\right.\right. \\
\left.\left.+\frac{\cos (\pi \cdot t)}{2}+7.5 \cdot \pi \cdot t\right)\right) \\
+\exp \left(j \cdot 2 \cdot \left(\cos (\pi \cdot t)+\frac{\cos (4 \cdot \pi \cdot t)}{2}\right.\right. \\
\left.\left.+\frac{\cos (2 \cdot \pi \cdot t)}{4}-9.5 \cdot \pi \cdot t\right)\right) .
\end{array}
$$

The time interval $t \in[-1,1]$ with the sampling rate $\Delta t=$ $2 / 128$ is used. Since the instantaneous frequency variations are very fast, the quadratic distributions $(N=2)$ are useless for its estimation. As an illustration, the Wigner distribution is given in Figure 1(a), while its smoothed and filtered version is given in Figure 1(b). Thus, in order to improve the results, the fourth-order distributions from the proposed class $\left(\mathrm{GCD}_{\mathrm{AF}}(t, \omega)\right.$ for $\left.N=4\right)$ are considered. The results obtained by using the Choi-Williams kernel, the Born-Jordan kernel, Sinc kernel, and Gaussian kernel $(\sigma=10)$ are shown in Figures 1(c)-1(f), respectively. Observe that the Gaussian kernel provides the efficient preservation of autoterms concentration, in addition to cross-terms suppression, and thus, provides slightly better results compared to other cases.

Additionally, the mean square errors (MSEs) are calculated as a quantitative measure for comparison, as follows:

$$
\text { MSE }=\frac{1}{N} \sum_{n=0}^{N-1}\left\{[f(n)-\bar{f}(n)]^{2}\right\},
$$




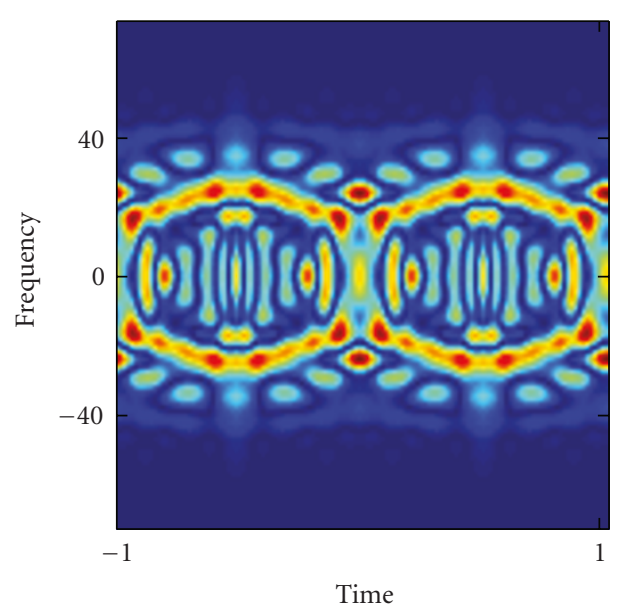

(a)

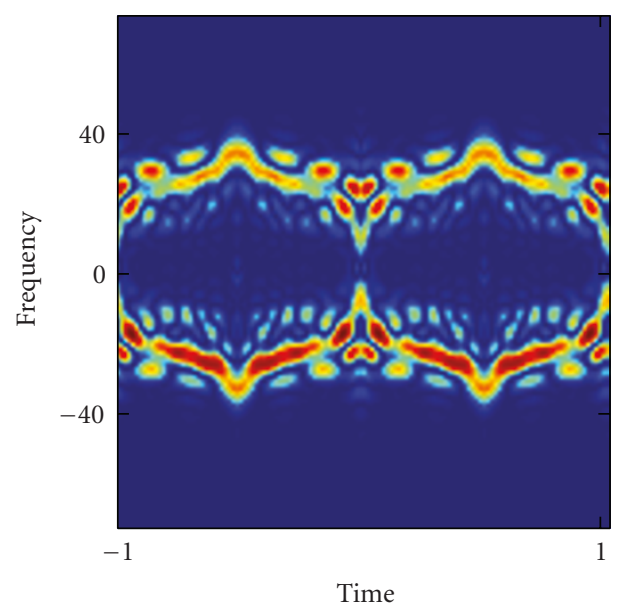

(c)

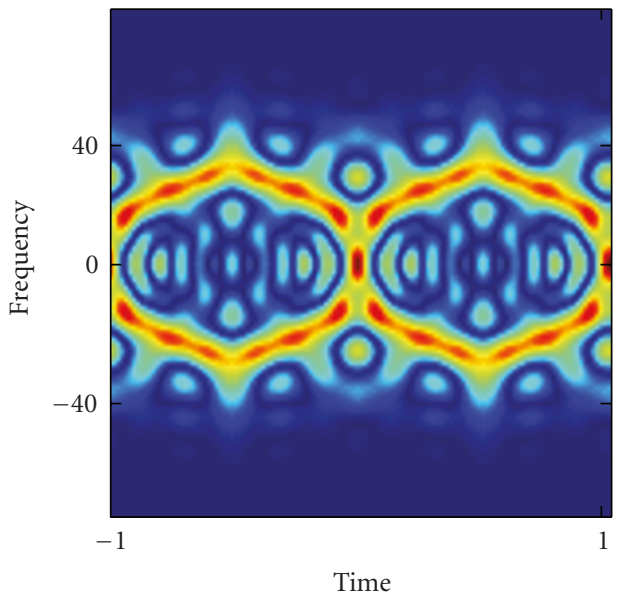

(b)

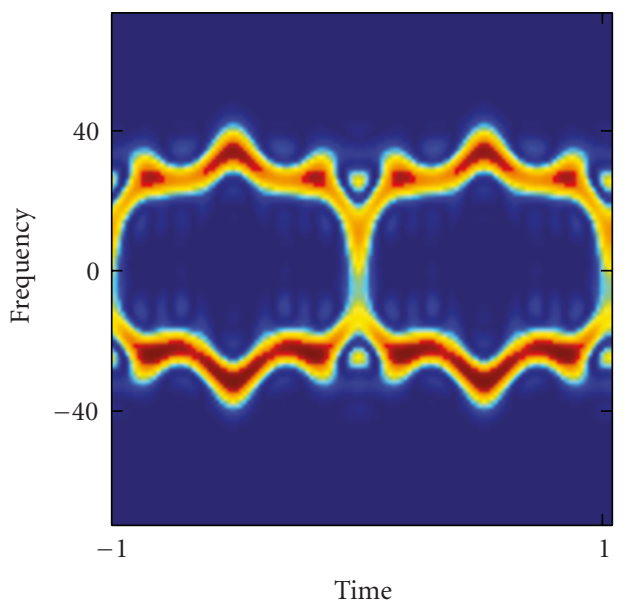

(d)

FIgUre 3: (a) Choi-Williams distribution; (b) $\mathrm{GCD}_{\mathrm{AF}}, N=2$ (smoothed pseudo-Wigner distribution); (c) $\mathrm{GCD}_{\mathrm{AF}}, N=4$ with Choi-Williams kernel; (d) $\mathrm{GCD}_{\mathrm{AF}}, N=4$ with Gaussian kernel.

where $f(n)$ represents the true instantaneous frequency while $\bar{f}(n)$ is the estimated instantaneous frequency by using the time-frequency distributions (TFD):

$$
\bar{f}(n)=\max _{m} \operatorname{TFD}(n, m) .
$$

The MSEs of IF estimations are shown in Table 2 for the following distributions: the Choi-Williams distribution, the smoothed pseudo Wigner distribution (these are special cases of $\mathrm{GCD}_{\mathrm{AF}}$ for $\left.N=2\right)$, and $\operatorname{GCD}_{\mathrm{AF}}(N=4)$ with: Choi-Williams kernel, Sinc kernel, Born-Jordan kernel, and Gaussian kernel. Note that the $\mathrm{GCD}_{\mathrm{AF}}(N=4)$ with Gaussian kernel provides the lowest MSE.

Furthermore, the considered signal $x(t)$ is corrupted by the white Gaussian noise, and the MSE of IF estimation is calculated in the presence of noise, as well. Several signalto-noise ratios (SNRs) are considered: $\mathrm{SNR}=30 \mathrm{~dB}, \mathrm{SNR}=$ $20 \mathrm{~dB}, \mathrm{SNR}=10 \mathrm{~dB}$, and $\mathrm{SNR}=5 \mathrm{~dB}$. The results are given in Table 3, for $\mathrm{GCD}_{\mathrm{AF}}$ with $N=4$ (Gaussian kernel), and compared with the results for Choi-Williams distribution.
Observe that for SNR $<10 \mathrm{~dB}$, the MSE for $\operatorname{GCD}_{\mathrm{AF}}(t, \omega)$ becomes significant.

Example 2. In order to illustrate the improvement of the distribution concentration by increasing distribution order $N$, we consider a multicomponent signal:

$$
\begin{gathered}
y(t)=\exp \left(j \cdot\left(3 \cos (\pi \cdot t)+\frac{2 \cos (7 \cdot \pi \cdot t)}{3}+11.5 \cdot \pi \cdot t\right)\right) \\
+\exp \left(2 \cdot j \cdot \left(\cos (\pi \cdot t)+\frac{\cos (2 \cdot \pi \cdot t)}{4}\right.\right. \\
\left.\left.+\frac{\cos (6 \cdot \pi \cdot t)}{2}-8.5 \cdot \pi \cdot t\right)\right) .
\end{gathered}
$$

The instantaneous frequency nonstationarity is enhanced in comparison with the previous example. The quadratic distribution $\left(\mathrm{GCD}_{\mathrm{AF}}\right.$ for $\left.N=2\right)$ obtained by using Gaussian kernel is shown in Figure 2(a). The fourth- $(N=4)$ and sixth- $(N=6)$ order distributions from the proposed class are shown in Figures 2(b) and 2(c), respectively (the same 


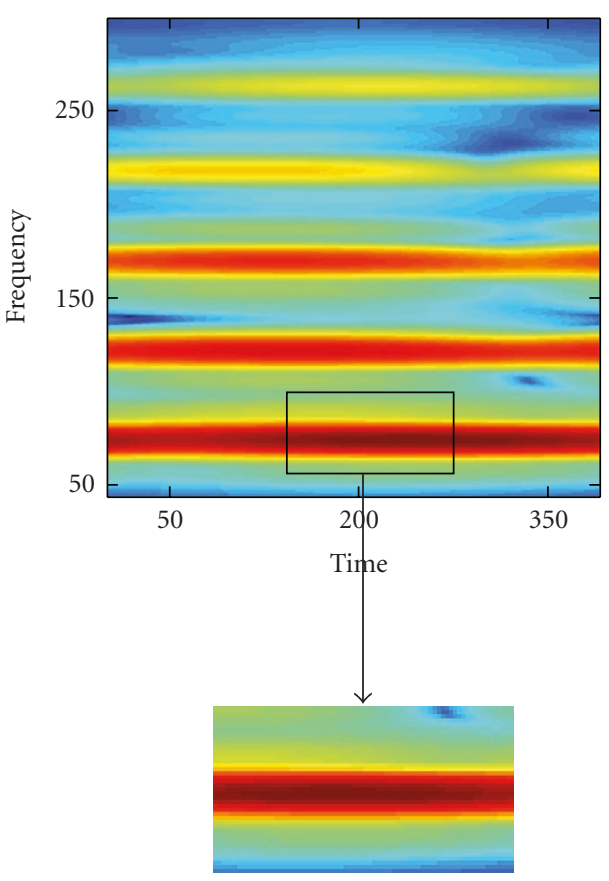

(a)

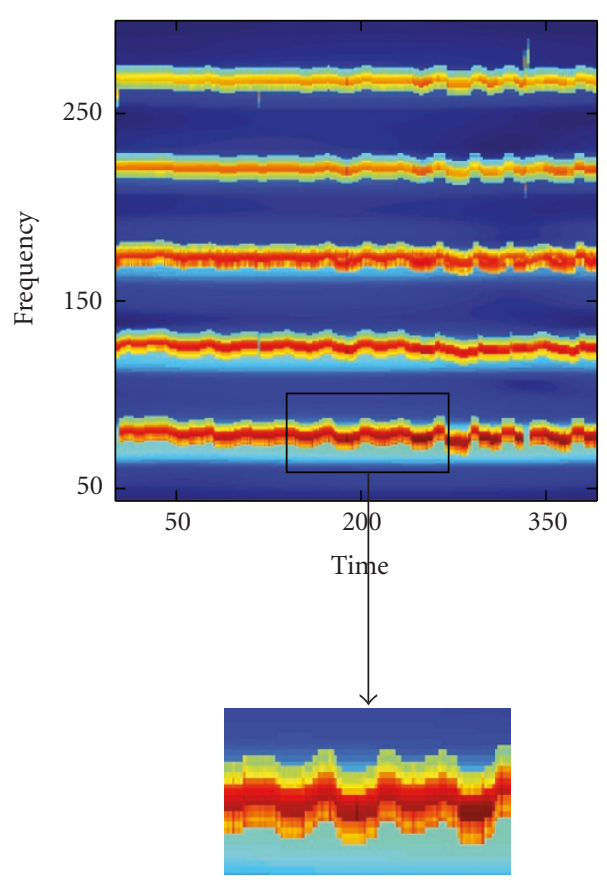

(b)

FIGURE 4: (a) Wigner distribution with Gaussian kernel and its zoomed region; (b) $\mathrm{GCD}_{\mathrm{AF}} N=4$, and its zoomed region for a real flute signal.

Gaussian kernel is used). It is obvious that the quadratic distribution does not follow the variations of instantaneous frequency. The results are quite improved for $N=4$. Further improvement is achieved by using the sixth order distribution $\left(\mathrm{GCD}_{\mathrm{AF}}\right.$ for $\left.N=6\right)$, that provides a very good representation of signal, producing higher concentration compared to the case $N=4$. Also, the inner interferences disappear. The exact and estimated instantaneous frequency (from $\mathrm{GCD}_{\mathrm{AF}}$ for $N=6$ ) is given in Figure 2(d).

Example 3. In the previous examples, we have considered signals whose components intersect in time domain. The analysis within the time-frequency plane becomes more complicated if signal components intersect in both time and frequency directions. Let us consider one such signal given by:

$z(t)=\cos \left(j \cdot\left(9 \cos (\pi \cdot t) \frac{2 \cos (3 \cdot \pi \cdot t)}{3}+\frac{5 \cos (5 \cdot \pi \cdot t)}{7}\right)\right)$

The Choi-Williams and Wigner distribution smoothed by using Gaussian kernel are shown in Figures 3(a), and 3(b), respectively. The fourth $\left(\mathrm{GCD}_{\mathrm{AF}}\right.$ for $\left.N=4\right)$ order distributions from the proposed class are calculated by using the Choi-Williams kernel (Figure 3(c)) and the Gaussian kernel (Figure 3(d)), respectively. Note that $\mathrm{GCD}_{\mathrm{AF}}$ for $N=4$ with Gaussian kernel performs better than the other considered distributions. The only problem in the IF estimation may appear at the cross-point of signal components.

Example 4. An additional test case is considered with a real multicomponent flute signal composed of several components, with nonlinear variations of the instantaneous frequency within the window. The results for Wigner distribution $(N=2)$ smoothed with Gaussian kernel and $\mathrm{GCD}_{\mathrm{AF}}$ for $N=4$ are presented in Figures $4(\mathrm{a})$ and $4(\mathrm{~b})$, respectively. Note that the $\mathrm{GCD}_{\mathrm{AF}}$ with $N=4$ provides significant improvements in the analysis of fast varying signal harmonics. Note that for quasistationary signals (such as speech), with minor variations of the instantaneous frequency within the window, the quadratic distributions provide sufficiently good results [28].

\section{Conclusion}

A class of time-frequency distributions with complex-lag argument, that provides arbitrary high concentration for multicomponent signals with fast varying instantaneous frequency, is proposed. It is based on new general form of ambiguity domain representation with complex-lag argument. Some of the existing fixed kernels are considered in the examples. It has been shown that the Gaussian smoothing kernel with low-pass characteristics provides very successful results. Future work could include the use of adaptable and signal-dependent kernels.

\section{Acknowledgments}

The authors are very thankful to anonymous reviewers for useful comments and suggestions that helped to improve the paper. This work is supported by the Montenegrin Ministry of Science and Montenegrin Agency for Telecommunications. 


\section{References}

[1] L. Cohen, "Time-frequency distributions-a review," Proceedings of the IEEE, vol. 77, no. 7, pp. 941-981, 1989.

[2] F. Hlawatsch and G. F. Boudreaux-Bartels, "Linear and quadratic time-frequency signal representations," IEEE Signal Processing Magazine, vol. 9, no. 2, pp. 21-67, 1992.

[3] LJ. Stankovic, "Multitime definition of the Wigner higher order distribution: L-Wigner distribution," IEEE Signal Processing Letters, vol. 1, no. 7, pp. 106-109, 1994.

[4] B. Barkat and B. Boashash, "Design of higher order polynomial Wigner-Ville distributions," IEEE Transactions on Signal Processing, vol. 47, no. 9, pp. 2608-2611, 1999.

[5] B. Boashash and B. Ristic, "Polynomial time-frequency distributions and time-varying higher order spectra: application to the analysis of multicomponent FM signals and to the treatment of multiplicative noise," Signal Processing, vol. 67, no. 1, pp. 1-23, 1998.

[6] G. Viswanath and T. V. Sreenivas, "IF estimation using higher order TFRs," Signal Processing, vol. 82, no. 2, pp. 127-132, 2002.

[7] R. L. Murray, A. Papandreou-Suppappola, and G. F. Boudreaux-Bartels, "New higher order spectra and timefrequency representations for dispersive signal analysis," in Proceedings of the IEEE International Conference on Acoustics, Speech, and Signal Processing (ICASSP '98), vol. 4, pp. 23052308, May 1998.

[8] I. Shafi, J. Ahmad, S. I. Shah, and F. M. Kashif, "Computing deblurred time-frequency distributions using artificial neural networks," Circuits, Systems, and Signal Processing, vol. 27, no. 3, pp. 277-294, 2008.

[9] I. Shafi, J. Ahmad, S. I. Shah, and F. M. Kashif, "Evolutionary time-frequency distributions using Bayesian regularised neural network model," IET Signal Processing, vol. 1, no. 2, pp. 97-106, 2007.

[10] P. Borgnat and P. Flandrin, "Time-frequency localization from sparsity constraints," in Proceedings of the IEEE International Conference on Acoustics, Speech, and Signal Processing (ICASSP '08), pp. 3785-3788, Las Vegas, Nev, USA, 2008.

[11] M. Jachan, G. Matz, and F. Hlawatsch, "Time-frequency ARMA models and parameter estimators for underspread nonstationary random processes," IEEE Transactions on Signal Processing, vol. 55, no. 9, pp. 4366-4381, 2007.

[12] S. Stanković and LJ. Stanković, "Introducing time-frequency distribution with a 'complex-time' argument," Electronics Letters, vol. 32, no. 14, pp. 1265-1267, 1996.

[13] LJ. Stanković, "Time-frequency distributions with complex argument,” IEEE Transactions on Signal Processing, vol. 50, no. 3, pp. 475-486, 2002.

[14] M. Morelande, B. Senadji, and B. Boashash, "Complexlag polynomial Wigner-Ville distribution," in Proceedings of the IEEE Speech and Image Technologies for Computing and Telecom, vol. 1, pp. 43-46, December 1997.

[15] C. Cornu, S. Stanković, C. Ioana, A. Quinquis, and LJ. Stanković, "Generalized representation of phase derivatives for regular signals," IEEE Transactions on Signal Processing, vol. 55, no. 10, pp. 4831-4838, 2007.

[16] S. Stanković, N. Žarić, I. Orović, and C. Ioana, "General form of time-frequency distribution with complex-lag argument," Electronics Letters, vol. 44, no. 11, pp. 699-701, 2008.

[17] H. Choi and W. J. Williams, "Improved time-frequency representation of multicomponent signals using exponential kernels," IEEE Transactions on Acoustics, Speech and Signal Processing, vol. 37, no. 6, pp. 862-871, 1989.
[18] Y. Zhao, L. E. Atlas, and R. J. Marks, "Use of cone-shaped kernels for generalized time-frequency representations of nonstationary signals," IEEE Transactions on Acoustics, Speech and Signal Processing, vol. 38, no. 7, pp. 1084-1091, 1990.

[19] LJ. Stanković, "Auto-term representation by the reduced interference distributions: a procedure for kernel design," IEEE Transactions on Signal Processing, vol. 44, no. 6, pp. 1557-1563, 1996.

[20] R. G. Baraniuk and D. L. Jones, "A signal-dependent timefrequency representation: optimal kernel design," IEEE Transactions on Signal Processing, vol. 41, no. 4, pp. 1589-1602, 1993.

[21] F. Hlawatsch and R. L. Urbanke, "Bilinear time-frequency representations of signals: the shift-scale invariant class," IEEE Transactions on Signal Processing, vol. 42, no. 2, pp. 357-366, 1994.

[22] R. G. Baraniuk and D. L. Jones, "Signal-dependent timefrequency analysis using a radially Gaussian kernel," Signal Processing, vol. 32, no. 3, pp. 263-284, 1993.

[23] M. G. Amin and W. J. Williams, "High spectral resolution time-frequency distribution kernels," IEEE Transactions on Signal Processing, vol. 46, no. 10, pp. 2796-2804, 1998.

[24] Y. Zhang, M. G. Amin, and G. J. Frazer, "High resolution timefrequency distributions for maneuvering target detection in over-the-horizon radars," in Proceedings of the IEEE International Conference on Acoustics, Speech and Signal Processing (ICASSP '03), vol. 6, pp. 437-440, April 2003.

[25] D. Boutana, B. Barkat, and F. Manir, "A proposed highresolution time-frequency distribution for multicomponent signal and speech signal," International Journal on Signal Processing, vol. 1, no. 2, pp. 167-170, 2004.

[26] M. J. Bastiaans, T. Alieva, and LJ. Stanković, "On rotated timefrequency kernels," IEEE Signal Processing Letters, vol. 9, no. 11, pp. 378-381, 2002.

[27] LJ. Stanković, "A method for time-frequency signal analysis," IEEE Transactions on Signal Processing, vol. 42, no. 1, pp. 225229, 1994.

[28] S. Stanković, "About time-variant filtering of speech signals with time-frequency distributions for hands-free telephone systems," Signal Processing, vol. 80, no. 9, pp. 1777-1785, 2000. 